Article

\title{
On the Origin of Morphological Structures of Planetary Nebulae
}

\author{
Sun Kwok ${ }^{1,2}$ (1) \\ 1 Department of Earth, Ocean, and Atmospheric Sciences, University of British Columbia, Vancouver, \\ BC V6T 1Z4, Canada; skwok@eoas.ubc.ca or sunkwok@hku.hk; Tel.: +1-778-858-5752 \\ 2 Laboratory for Space Research, The University of Hong Kong, Hong Kong, China
}

Received: 24 May 2018; Accepted: 22 June 2018; Published: 26 June 2018

\begin{abstract}
We suggest that most of the mass in planetary nebulae $(\mathrm{PNe})$ resides in the equatorial region and the spherical envelope and the optically bright lobes of $\mathrm{PNe}$ are in fact low-density cavities cleared out by fast outflows and photoionized by UV photons leaked from the torus. The nature of multi-polar PNe is discussed under this framework.
\end{abstract}

Keywords: planetary nebulae; asymptotic giant branch stars; mass loss

\section{Introduction}

The diverse morphological shapes of planetary nebulae $(\mathrm{PNe})$ were recognized since the beginning of PNe research [1]. Does this diversity suggest that every PN is different, or is there an intrinsic structure common to all PNe? The possibility that there is a uniform 3-D structure of PNe was explored by models such as an open-ended toroid [2] or an ellipsoidal shell [3] inclined w.r.t. the line of sight. Ionization penetrating to different depths could create the different apparent shapes of PNe [4].

A separate question is what is the physical mechanism that creates such non-spherically symmetric structures? The realization that $\mathrm{PNe}$ are formed by the process of interacting winds [5] led to the suggestion that nebular shaping can be achieved by asymmetry in one or more of the stellar winds [6].

Observations with CCD cameras, space-based observations, and imaging beyond the visible wavelengths, have greatly expanded our capabilities of mapping the morphological structures of PN. In this paper, we discuss whether these new observations can lead to a new paradigm in our understanding of the origin of morphological structures of PNe.

\section{Problems with Morphological Classifications}

Our morphological classifications of PNe are primarily based on the apparent morphological shapes of optical images of PNe. These classification schemes suffer from the following problems:

(i). Orientation effects (PNe are 3D but images are 2D). A near-pole-on bipolar nebula may appear as a ring because of the prominence of the torus. Many well-known PNe (the Ring, the Helix, the Dumbbell, NGC 7027) turn out to have similar intrinsic bipolar structures [7-10].

(ii). Dynamic range: if we go deep enough we see more/different structures. The well-known Messier PN M76 turned out to be bipolar when observed by CCD detectors, when earlier photographic plate images only showed the central torus. The bipolar lobes of Sh 1-89 were only found with narrow-band CCD imaging [11].

(iii). Limited field of view (FoV). We may miss large outer structures because the limited FoV of observations (examples: IPHAS PN-1 [12], M1-41 [13]). 
(iv). limited wavelength coverage. Bipolar structures not obvious in visible images may reveal themselves in the infrared [14].

(v). Internal dust extinction. Optical morphology of PNe may be affected by effects of circumstellar dust extinction [15].

\section{Multipolar Nebulae}

As the result of high dynamic-range imaging, more and more PNe are found to be multipolar [16, 17]. HST observations have found that many compact PNe have multi-polar lobes [18]. Interestingly, many of the multi-polar lobes are roughly equal in length $[19,20]$. Some PNe with prominent bipolar lobes also show secondary multi-polar lobes in other directions [21]. The existence of multi-polar nebulae suggests collimated ejections formed simultaneously or episodically [18]. Precessing bipolar, rotating episodic jets have been proposed to explain the observed morphology [22].

What is the fraction of multi-polar PNe among the PNe population? Current statistics suggests a range between 10-20\% [23,24], but these are almost certainly lower limits as high-dynamic-range imaging of PNe has only been done for a small fraction of PNe. If a majority of PNe are multi-polar, what are the consequences? We have performed an exercise assuming that PNe have 3 pairs of identical-length lobes oriented at random directions and simulate their apparent images when observed from different lines of sight to different degrees of sensitivity. We found that many different observed morphological shapes of PNe can be reproduced with this simulation. For example, tori and double inner-outer bipolar lobes are the result of overlapping multi-polar lobes, and ansae and knots may be the bright tips of undetected lobes [25]. Nearly aligned pairs of lobes can give the appearance of point-symmetric S-shape morphology, which does not necessarily imply precession. It is remarkable that morphology of very diverse PNe can be simulated by a single, very simple 3-D model when orientation and sensitivity effects are taken into account.

In the past, we have relied on slit spectroscopy to infer the kinematic structure of PNe. The best way to test the multipolar hypothesis is through integral field spectroscopy. The velocity maps from such observations can be compared with 3-D spatial-kinematic models to determine the true intrinsic structure of PNe.

\section{Unseen "Dark" Matter in Planetary Nebulae}

Many bipolar PNe (e.g., NGC 6302, NGC 2346) have very tight waists, suggesting that they are being confined by unseen external material. Other bipolar PNe (e.g., IC 4406, Hen 3-401, Hen 2-320) show sharp, well-defined lobe boundaries, suggesting that the optical lobes are dynamically confined by external media (Figure 1). This unseen matter is probably in the form of molecular gas and can be traced by molecular-line imaging. Mapping of the $2.12 \mu \mathrm{m} \mathrm{H}_{2}$ line requires the molecule to be highly excited by shocks or UV radiation so this line can map only dynamically interacting regions but is not a good tracer of cold molecular gas. The $\mathrm{H}_{2}$ ground-state rotational transition (para $\mathrm{S}(0) \mathrm{J}=2-0$ ) requires less $(500 \mathrm{~K})$ excitation but this $28 \mu \mathrm{m}$ line cannot be observed from the ground [26].

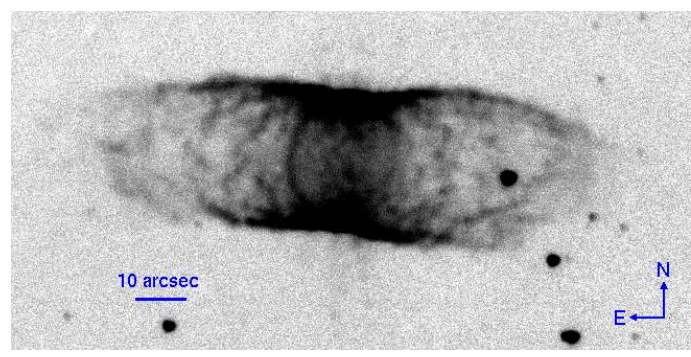

Figure 1. Continuum-subtracted $\mathrm{H}_{2} 2.12 \mu \mathrm{m}$ line image of IC 4406 obtained with the Canada-France-Hawaii Telescope. The bipolar lobes have sharp boundaries and are clearly confined by an external medium. 
Current CO line mapping from $\mathrm{mm} /$ submm-wave interferometers such as SMA and ALMA mainly reveals the molecular torus around the waist of bipolar nebulae [27,28]. Mapping of the extended molecular gas will require higher surface brightness sensitivity observations.

The dust mixed with the cold molecular gas is probably too low temperature to be observed by mid-infrared (10-20 $\mu \mathrm{m})$ imaging. Current mid-infrared imaging of PNe mainly reveals dust in the torus or in the bipolar lobes $[29,30]$. Although efforts have been made to map the cold dust component by Spitzer [31,32], AKARI [33], and Herschel [34-36], the extended cold dust component be truly revealed with high angular resolution, high sensitivity, far infrared imaging with facilities such as SOFIA.

\section{Discussion}

Many of the present theories on the origin of morphological structures of PNe are based on the assumption that the observed bright nebulosity represents ejection of physical matter. However, the visible brightness of $\mathrm{PNe}$ is due to recombination lines of $\mathrm{H}$ and $\mathrm{He}$ and collisionally excited lines of metals, all strong emissions originating from the ionized region of PNe that only represents a small fraction of the total mass of PNe. We suggest that the bipolar lobes of PNe are not regions of massive ejection, but low-density cavities carved out of the neutral circumstellar envelope [22]. In the AGB stage, a massive circumstellar envelope is created by mass loss via a slow wind. In the post-AGB phase, collimated fast outflows, energetic but not massive, emerge from the central star. These outflows break through less dense regions of the circumstellar torus, creating cavities in the envelope. Dust scattering of visible photons from the central star leads to bipolar and multi-polar nebulosity observed in proto-PNe [37]. In the subsequent PNe phase, UV photons from the now hot central star ionize the low-density cavity, illuminating the bipolar region. Optical morphology of PN is therefore not defined by regions of massive matter ejection, but represents holes in the matter distribution where densities are low enough for UV to ionize. The observable effects of a collimated fast wind interacting with clumpy circumstellar envelope have been explored by Steffen et al. [38].

As central stars of PNe evolve to higher temperatures, their radii contract and the escape velocities increase. These effects lead to higher power fast winds, hotter shocked bubbles, and higher thermal pressure [39]. The dynamical effects of interacting winds may wash out the multi-polar structures observed in younger PNe, leading to a lower frequency of multi-polar structures in evolved PNe.

As PNe evolve and densities drop, the ionization front will eventually breakthrough and the nebulae becomes density bounded. The morphology of the PNe will also become more spherical. The low-surface-brightness spherically-shaped nebulae found in deep H $\alpha$ surveys $[40,41]$ are probably examples of density-bounded, highly evolved PNe. The fraction of PNe that is bipolar is therefore dependent on age.

\section{Conclusions}

Recent high-dynamic-range optical and infrared observations have revealed that many $\mathrm{PNe}$ previously classified as round or elliptical are in fact bipolar. Statistical analysis of morphological classes based on apparent shapes is therefore not reliable and the true 3-D structures PNe can only be found by proper modeling of the brightness and kinematic structures. We suggest that multipolar nebulae are much more common than currently believed and more PNe will show multi-polar structure with deeper imaging. Some of the morphological features such as tori, filaments, knots, and ansae could be manifestation of multi-polar nature of the objects. The optical lobes of bipolar/multipolar PNe and proto-PNe are not volumes of concentrated mass but low-density cavities ionized or illuminated by the central star. The true mass distribution of PNe can only be revealed with future far infrared and $\mathrm{mm} /$ submm molecular-line imaging.

Funding: This work is supported by grants from the Research Grants Council of Hong Kong and the Natural Sciences and Engineering Research Council of Canada.

Acknowledgments: S.K. thanks Alberto López for valuable comments on an earlier version of this paper. 
Conflicts of Interest: The author declares no conflict of interest.

\section{References}

1. Curtis, H.D. The Planetary Nebulae. Publ. Lick Obs. 1918, 13, 55-74.

2. Khromov, G.S.; Kohoutek, L. Morphological Study of Planetary Nebulae. In Symposium-International Astronomical Union; Cambridge University Press: Cambridge, UK, 1968; Volume 34, p. 227.

3. Masson, C.R. On the Structure of Ionization-Bounded Planetary-Nebulae. Astrophys. J. 1990, 348, 580-587. [CrossRef]

4. Zhang, C.Y.; Kwok, S. A Morphological Study of Planetary Nebulae. Astrophys. J. Suppl. Ser. 1998, $117,341$. [CrossRef]

5. Kwok, S. From red giants to planetary nebulae. Astrophys. J. 1982, 258, 280-288. [CrossRef]

6. Balick, B. The evolution of planetary nebulae. I-Structures, ionizations, and morphological sequences. Astron. J. 1987, 94, 671-678. [CrossRef]

7. Bryce, M.; Balick, B.; Meaburn, J. Investigating the Haloes of Planetary Nebulae-Part Four-NGC6720 the Ring Nebula. Mon. Not. R. Astron. Soc. 1994, 266, 721-732. [CrossRef]

8. Meaburn, J.; Boumis, P.; López, J.A.; Harman, D.J.; Bryce, M.; Redman, M.P.; Mavromatakis, F. The creation of the Helix planetary nebula (NGC 7293) by multiple events. Mon. Not. R. Astron. Soc. 2005, 360, 963-973. [CrossRef]

9. Kwok, S.; Chong, S.-N.; Koning, N.; Hua, T.; Yan, C.-H. The True Shapes of the Dumbbell and the Ring. Astrophys. J. 2008, 689, 219-224. [CrossRef]

10. Latter, W.B.; Dayal, A.; Bieging, J.H.; Meakin, C.; Hora, J.L.; Kelly, D.M.; Tielens, A.G.G.M. Revealing the Photodissociation Region: HST/NICMOS Imaging of NGC 7027. Astrophys. J. 2000, 539, 783-797. [CrossRef]

11. Hua, C.T. Deep morphologies of type I planetary nebulae. Astron. Astrophys. Suppl. Ser. 1997, 125, 355-366. [CrossRef]

12. Mampaso, A.; Corradi, R.L.; Viironen, K.; Leisy, P.; Greimel, R.; Drew, J.E.; Barlow, M.J.; Frew, D.J.; Irwin, J.; Morris, R.A.H.; et al. The "Príncipes de Asturias" nebula: A new quadrupolar planetary nebula from the IPHAS survey. Astron. Astrophys. 2006, 458, 203-212. [CrossRef]

13. Zhang, Y.; Hsia, C.-H.; Kwok, S. Planetary Nebulae Detected in the Spitzer Space Telescope GLIMPSE 3D Legacy Survey. Astrophys. J. 2012, 745, 59. [CrossRef]

14. Zhang, Y.; Kwok, S. Planetary Nebulae Detected in the Spitzer Space Telescope GLIMPSE II Legacy Survey. Astrophys. J. 2009, 706, 252-305. [CrossRef]

15. Lee, T.-H.; Kwok, S. Dust Extinction in Compact Planetary Nebulae. Astrophys. J. 2005, 632, 340-354. [CrossRef]

16. Manchado, A.; Stanghellini, L.; Guerrero, M.A. Quadrupolar Planetary Nebulae: A New Morphological Class. Astrophys. J. 1996, 466, L95. [CrossRef]

17. Lopez, J.A.; Meaburn, J.; Bryce, M.; Holloway, A.J. The Morphology and Kinematics of the Complex Polypolar Planetary Nebula NGC 2440. Astrophys. J. 1998, 493, 803-810. [CrossRef]

18. Hsia, C.-H.; Chau, W.; Zhang, Y.; Kwok, S. Hubble Space Telescope Observations and Geometric Models of Compact Multipolar Planetary Nebulae. Astrophys. J. 2014, 787, 25. [CrossRef]

19. Sahai, R. The starfish twins: Two young planetary nebulae with extreme multipolar morphology. Astrophys. J. 2000, 537, L43-L47. [CrossRef]

20. Sahai, R.; Nyman, L.-Å.; Wootten, A. HE 2-113: A Multipolar Planetary Nebula with Rings around a Cool Wolf-Rayet Star. Astrophys. J. 2000, 543, 880-888. [CrossRef]

21. López, J.A.; García-Díaz, M.T.; Steffen, W.; Riesgo, H.; Richer, M.G. Morpho-kinematic Analysis of the Point-symmetric, Bipolar Planetary Nebulae $\mathrm{Hb} 5$ and K 3-17, A Pathway to Poly-polarity. Astrophys. J. 2012, 750, 131. [CrossRef]

22. Lopez, J.A.; Vazquez, R.; Rodriguez, L.F. The Discovery of a Bipolar, Rotating, Episodic Jet (BRET) in the Planetary Nebula KjPn 8. Astrophys. J. 1995, 455, L63-L66. [CrossRef]

23. Manchado, A.; García-Hernández, D.A.; Villaver, E.; de Massas, J.G. Morphological Classification of Post-AGB Stars. In Why Galaxies Care about AGB Stars II: Shining Examples and Common Inhabitants; Kerschbaum, F., Ed.; Astronomical Society of the Pacific: San Francisco, CA, USA, 2011; p. 161. 
24. Sahai, R.; Morris, M.R.; Villar, G.G. Young Planetary Nebulae: Hubble Space Telescope Imaging and a New Morphological Classification System. Astron. J. 2011, 141, 134. [CrossRef]

25. Chong, S.-N.; Kwok, S.; Imai, H.; Tafoya, D.; Chibueze, J. Multipolar Planetary Nebulae: Not as Geometrically Diversified as Thought. Astrophys. J. 2012, 760, 115. [CrossRef]

26. Kwok, S. Physics and Chemistry of the Interstellar Medium; University Science Books; Cambridge University Press: Cambridge, UK, 2006.

27. Trung, D.-V.; Bujarrabal, V.; Castro-Carrizo, A.; Lim, J.; Kwok, S. Massive Expanding Torus and Fast Outflow in Planetary Nebula NGC 6302. Astrophys. J. 2008, 673, 934-941. [CrossRef]

28. Santander-García, M.; Bujarrabal, V.; Alcolea, J.; Castro-Carrizo, A.; Contreras, C.S.; Quintana-Lacaci, G.; Corradi, R.L.M.; Neri, R. ALMA high spatial resolution observations of the dense molecular region of NGC 6302. Astron. Astrophys. 2017, 597, A27. [CrossRef] [PubMed]

29. Muthumariappan, C.; Kwok, S.; Volk, K. Subarcsecond Mid-Infrared Imaging of Dust in the Bipolar Nebula Hen 3-401. Astrophys. J. 2006, 640, 353-359. [CrossRef]

30. Volk, K.; Hrivnak, B.J.; Su, K.Y.L.; Kwok, S. An Infrared Imaging Study of the Bipolar Proto-Planetary Nebula IRAS 16594-4656. Astrophys. J. 2006, 651, 294-300. [CrossRef]

31. Su, K.Y.L.; Kelly, D.M.; Latter, W.B.; Misselt, K.A.; Frank, A.; Volk, K.; Engelbracht, C.W.; Gordon, K.D.; Hines, D.C.; Morrison, J.E.; et al. High spatial resolution mid- and far-infrared imaging study of NGC 2346. Astrophys. J. Suppl. Ser. 2004, 154, 302-308. [CrossRef]

32. Ueta, T. Spitzer MIPS Imaging of NGC 650: Probing the History of Mass Loss on the Asymptotic Giant Branch. Astrophys. J. 2006, 650, 228-236. [CrossRef]

33. Izumiura, H.; Ueta, T.; Yamamura, I.; Nakada, Y.; Matsunaga, N.; Ita, Y.; Matsuura, M.; Fukushi, H.; Mito, H.; Tanabe, T. Far-IR Imaging Survey of the Extended Dust Shells of Evolved Stars with AKARI. In AKARI, a Light to Illuminate the Misty Universe; Onaka, T., Ed.; Astronomical Society of the Pacific: San Francisco, CA, USA, 2009; p. 127.

34. Ueta, T.; Ladjal, D.; Exter, K.M.; Otsuka, M.; Szczerba, R.; Siódmiak, N.; Aleman, I.; van Hoof, P.A.M.; Kastner, J.H.; Montez, R.; et al. The Herschel Planetary Nebula Survey (HerPlaNS)-I. Data overview and analysis demonstration with NGC 6781. Astron. Astrophys. 2014, 565, A36. [CrossRef]

35. Van Hoof, P.A.M.; Van de Steene, G.C.; Exter, K.M.; Barlow, M.J.; Ueta, T.; Groenewegen, M.A.T.; Gear, W.K.; Gomez, H.L.; Hargrave, P.C.; Ivison, R.J.; et al. A Herschel study of NGC 650. Astron. Astrophys. 2013, 560, A7. [CrossRef]

36. Van de Steene, G.C.; van Hoof, P.A.M.; Exter, K.M.; Barlow, M.J.; Cernicharo, J.; Etxaluze, M.; Gear, W.K.; Goicoechea, J.R.; Gomez, H.L.; Groenewegen, M.A.T.; et al. Herschel imaging of the dust in the Helix nebula (NGC 7293). Astron. Astrophys. 2015, 574, A134. [CrossRef]

37. Koning, N.; Kwok, S.; Steffen, W. Post Asymptotic Giant Branch Bipolar Reflection Nebulae: Result of Dynamical Ejection or Selective Illumination? Astrophys. J. 2013, 765, 92. [CrossRef]

38. Steffen, W.; Koning, N.; Esquivel, A.; García-Segura, G.; García-Díaz, M.T.; López, J.A.; Magnor, M. A wind-shell interaction model for multipolar planetary nebulae. Mon. Not. R. Astron. Soc. 2013, 436, 470-478. [CrossRef]

39. Schönberner, D.; Jacob, R.; Lehmann, H.; Hildebrandt, G.; Steffen, M.; Zwanzig, A.; Sandin, C.; Corradi, R.L.M. A hydrodynamical study of multiple-shell planetary nebulae. III. Expansion properties and internal kinematics: Theory versus observation. Astron. Nachr. 2014, 335, 378-408. [CrossRef]

40. Parker, Q.A.; Phillipps, S.; Pierce, M.J.; Hartley, M.; Hambly, N.C.; Read, M.A.; MacGillivray, H.T.; Tritton, S.B.; Cass, C.P.; Cannon, R.D.; et al. The AAO/UKST superCOSMOS H $\alpha$ survey. Mon. Not. R. Astr. Soc. 2005, 362, 689-710. [CrossRef]

41. Parker, Q.A.; Acker, A.; Frew, D.J.; Hartley, M.; Peyaud, A.E.J.; Ochsenbein, F.; Phillipps, S.; Russeil, D.; Beaulieu, S.F.; Cohen, M.; et al. The Macquarie/AAO/Strasbourg H $\alpha$ planetary nebula catalogue: MASH. Mon. Not. R. Astr. Soc. 2006, 373, 79-94. [CrossRef]

(c) 2018 by the author. Licensee MDPI, Basel, Switzerland. This article is an open access article distributed under the terms and conditions of the Creative Commons Attribution (CC BY) license (http:/ / creativecommons.org/licenses/by/4.0/). 